\title{
Signaling for Lymphangiogenesis via VEGFR-3 is Required for the Early Events of Metastasis
}

\author{
Masataka Matsumoto, ${ }^{1,5}$ Sally Roufail, ${ }^{1,4}$ Rachael Inder, ${ }^{1}$ Carol Caesar, ${ }^{1,4}$ Tara \\ Karnezis, ${ }^{1,4}$ Ramin Shayan, ${ }^{1,2,4}$ Rae H. Farnsworth, ${ }^{1,2,4}$, Teruhiko Sato ${ }^{15}$, , Marc G. \\ Achen, ${ }^{1,4,5}$ G. Bruce Mann ${ }^{2,3}$, Steven A. Stacker ${ }^{1,4,5^{*}}$
}

${ }^{1}$ Ludwig Institute for Cancer Research, Post Office Box 2008, Royal Melbourne Hospital, Parkville, Victoria 3050, Australia; ${ }^{2}$ Department of Surgery, Royal Melbourne Hospital, University of Melbourne, Parkville, Victoria 3050, Australia; ${ }^{3}$ Royal Womens Hospital, Parkville, Victoria 3052, Australia, ${ }^{4}$ Tumour Angiogenesis Program, Peter MacCallum Cancer Centre, East Melbourne, Victoria 8006, Australia, ${ }^{6}$ Sir Peter MacCallum Department of Oncology, The University of Melbourne, Parkville, Victoria 3010, Australia

*Correspondence and reprint requests to: Steven A. Stacker, Peter MacCallum Cancer Centre, Locked bag 1, A'Beckett Street, Melbourne, Victoria 8006, AUSTRALIA; E-mail: steven.stacker@petermac.org; Phone: +613-9656-5263; Fax:+613-9656-1411

${ }^{5}$ Current address: For MM: Department of Surgical Oncology and Digestive Surgery, Field of Oncology, Course of Advanced Therapeutics, Graduate School of Medical and Dental Sciences, Kagoshima University, 8-35-1, Sakuragaoka, Kagoshima 890-8520, Japan. For TS: Department of Respiratory Medicine, School of Medicine, Juntendo University, 2-1-1 Hongo, Bunkyo-Ku, Tokyo, 113-8421, Japan.

This work was supported by Program Grants and Research Fellowships from the National Health and Medical Research Council of Australia and by funds from the Operational Infrastructure Support Program, Victorian Government, Australia. Affiliations: SAS and MGA are on the Scientific Advisory Board of Vegenics Ltd, a wholly owned subsidiary of Circadian Technologies, are stock holders and consultants.

Running Head: VEGFR-3 in early stages of metastasis

Keywords: lymphatic vessels, lymph nodes, angiogenesis, breast cancer 


\section{Non-standard abbreviations:}

LN, lymph node; LVD, lymphatic vessel density; LYVE-1 Lymphatic Vessel Endothelial Receptor 1; NK, natural killer; Mab, monoclonal antibody; MTT 3-[4,5-dimethylthiazol-2-yl]-2,5 diphenyl tetrazolium bromide; PBS phosphate-buffered saline; PECAM-1, Platelet Endothelial Cell Adhesion Molecule-1; SCID/NOD, severe combined immunodeficiency/non-obese diabetic; VEGF, vascular endothelial growth factor; VEGFR, vascular endothelial growth factor receptor. 


\begin{abstract}
Metastasis to regional lymph nodes is an important and early event in many tumors. Vascular endothelial growth factor-C (VEGF-C), VEGF-D and their receptor VEGFR-3, play a role in tumor spread via the lymphatics, although the timing of their involvement is not understood. In contrast, VEGFR-2, activated by VEGF-A, VEGF-C and VEGF-D, is a mediator of angiogenesis and drives primary tumor growth. We demonstrate the critical role for VEGFR-3, but not VEGFR-2, in the early events of metastasis. In a tumor model exhibiting both VEGF-D-dependent angiogenesis and lymphangiogenesis, an antibody to VEGFR-2 (DC101) was capable of inhibiting angiogenesis (79\% reduction in PECAM+ blood vessels) and growth (93\% reduction in tumor volume). However, unlike an anti-VEGFR-3 Mab (mF4-31C1), DC101 was not capable of eliminating either tumor lymphangiogenesis or lymphogenous metastasis $(60 \%$ reduction of lymph node metastasis by DC101 vs $95 \%$ by mF4-31C1). Early excision of the primary tumors demonstrated that VEGF-D-mediated tumor spread precedes angiogenesis-induced growth. Small but highly metastatic primary human breast cancers had significantly higher lymphatic vessel density $\left(23.1\right.$ vessels $\left./ \mathrm{mm}^{2}\right)$ than size-matched (11.7) or larger non-metastatic tumors (12.4) thus supporting the importance of lymphatic vessels, as opposed to angiogenesis-mediated primary tumor growth, for nodal metastasis. These results suggest that lymphangiogenesis via VEGF-D is more critical than angiogenesis for nodal metastasis.
\end{abstract}




\section{Introduction}

The natural history of most epithelial malignancies involves an initial phase of proliferation of cancer cells at the site or origin, followed by metastasis to a variety of possible destinations. Involvement of regional lymph nodes is often a relatively early event, and is an important prognostic factor in most cancers [1]. Malignant cells may metastasise via lymphatic or blood vessels, but it is unclear which vessel types are involved in early metastatic events. Further, the relationship between nodal metastasis and systemic metastasis is uncertain, although recent genomic sequencing of cancers suggests a capacity for clonal evolution of metastasis, with individual metastatic lesions able to seed further metastasis[2].

The study of tumor angiogenesis has revealed the key role that growth factors such as VEGF-A play in mediating the proliferation of tumor-associated blood vessels [3, 4]. Further, anti-angiogenic treatment of these vessels using inhibitors of VEGF and VEGFR-2 can reduce tumor angiogenesis and slow the rate of tumor growth in both experimental models and in established human tumors[5-7]. However, clinical trials using an anti-VEGF-A monoclonal antibody (Avastin/Bevacizumab) as adjuvant therapy in early-stage colon cancer failed to demonstrate an improved disease-free survival [8]. An interpretation of these outcomes, in terms of the biology of the primary tumor, is that while an anti-VEGF-A approach is effective for controlling primary tumor growth, other signaling pathways contribute to tumor dissemination.

The discovery of lymphangiogenic growth factors such as VEGF-C[9] and VEGF-D[10, 11], and of lymphatic markers such as LYVE-1[12] and podoplanin[13], has accelerated 
the study of tumor lymphangiogenesis, and the role of lymphatic vessels in cancer[14-19]. Approaches driving expression of these growth factors in mouse tumor models have demonstrated their potency at promoting both tumor angiogenesis and lymphagiogenesis, and lymph node and distant organ metastasis[14, 18, 20-22]. In a VEGF-D-positive tumor model, lymphatic metastasis can be inhibited by VEGF-D-specific monoclonal antibodies (Mabs) [18, 23]. Further, expression of VEGF-C and VEGF-D in numerous human tumors has shown a correlation with lymph node metastasis, suggesting potential utility of these molecules as prognostic factors and as therapeutic targets [24-34].

In the present study we use a variety of mouse tumor models and neutralizing antibodies specific for VEGFR-2 and VEGFR-3 to address the contribution of VEGF-D-dependent lymphangiogensis and angiogenesis in the initial phase of tumor metastasis. Our results show that signaling through VEGFR-3 is required for the initial steps of VEGF-D-mediated lymphogenous metastasis, and that lymphogenous metastasis is less dependent on enhanced angiogenesis mediated through VEGFR-2.

\section{Materials and Methods}

\section{Experimental Animals}

We used female SCID/NOD mice (Mus musclus), which have deficient NK-cell function (ARC and Walter and Eliza Hall Institute for Medical Research, Australia), 8-12 weeks of age. All experiments performed on animals were in accordance with guidelines of the Regional Animal Ethics Committee set by the National Health and Medical Research Council of Australia. 


\section{Tumor Cell Lines and Cultures}

The 293-VEGF-D cell line (293; human embryonic kidney cells) expressing full-length human VEGF-D cDNA with amino-terminal FLAG sequence (VEGF-D-FULL-N-FLAG) via an pAPEX-3 expression vector was used to generate subcutaneous tumors expressing the growth factor VEGF-D as previously described[18]. A 293EBNA line stably transfected with APEX-3 vector alone (293-APEX) was used as a control. Cell lines were maintained in Dulbecco's modified Eagle's medium with 10\% FBS, $5 \mathrm{mM}$ L-glutamine, $50 \mu \mathrm{g} / \mathrm{ml}$ gentamicin and $100 \mu \mathrm{g} / \mathrm{ml}$ of hygromycin in a humidified atmosphere of $10 \% \mathrm{CO}_{2}$. The growth rates of cell lines in vitro were not statistically different as assayed with MTT. VEGF-D was bioactive as assessed by VEGFR-2 and VEGFR-3 bioassays[23, 35].

\section{Anti-VEGFR-2, Anti-VEGFR-3 and Anti-VEGF-D Monoclonal Antibodies}

DC101 and mF4-31C1 are neutralizing rat monoclonal antibodies directed against mouse VEGFR-2 and VEGFR-3, respectively, that were supplied by ImClone Systems Incorporated (New York, NY). The mF4-31C1 antibody prevents lymphangiogenesis in adult mice without effects on either angiogenesis or the survival or function of existing lymphatic vessels[36]. The VD1 anti-VEGF-D monoclonal antibody was produced essentially as described previously[23] - it was purified from the culture media of the VD1 hybridoma cell line by affinity chromatography with protein G-Sepharose (Amersham Pharmacia Biotech) according to the manufacturer. All antibodies were injected in PBS buffers.

\section{3-VEGF-D Xenograft Model}

The 293-VEGF-D tumor model, in which xenografts of the VEGF-D-secreting 
transfected 293EBNA-1 tumor cell line are implanted subcutaneously into the skin of SCID/NODmice, has been previously described[18]. Control tumors which express only the pAPEX vector with no VEGF-D-encoding insert (designated 293-APEX) grow less rapidly and show no spread to regional lymph nodes. The 293-VEGF-D tumors produce full-length VEGF-D polypeptide that is proteolytically processed to generate mature VEGF-D which is capable of inducing both tumor angiogenesis and lymphangiogenesis, and driving lymphogenous spread to regional lymph nodes[18]. Here, mice inoculated with 293-VEGF-D or control 293-APEX cells were divided into six treatment groups ( $n=10$ per group). Mice injected with VEGF-D-expressing tumor cells were treated with vehicle alone, VD1, mF4-31C1 or DC101 monoclonal antibodies (Mabs) or the combination of mF4-31C1 and DC101. The negative control tumor, 293-APEX, was also used. The mice were anesthetized with inhalation of nitrous oxide and 2-chloro-2-difluoromethoxy-1,1,1-trifluoro-ethane (isoflurane), and $2.5 \times 10^{7}$ cells were injected on the left flank of each mouse subcutaneously. Antibodies were injected intraperitoneally and treatment started the day after tumors were injected. The VD1 antibody was injected at 400-800 $\mu \mathrm{g} /$ mouse twice a week (approx 20-40 mg/kg), and DC101 and mF4-31C1were injected at 700-800 $\mu \mathrm{g} / \mathrm{mouse}$ (approx 30-37.5 mg/kg) and $1 \mathrm{mg} / \mathrm{mouse}(40-50 \mathrm{mg} / \mathrm{kg})$ three times a week, respectively. The resulting tumors were measured with digital calipers. Tumor volumes were calculated as follows: volume $=$ length $\times$ width ${ }^{2} \times 0.52$. Four to five weeks later the mice were sacrificed to harvest the primary tumor, surrounding tissue and regional lymph nodes. In a variation of the model, established primary tumors were surgically excised on day 7 after injection of the tumor cells. Wound edges were approximated with surgical clips and the wound monitored for five weeks. Mice with a local recurrence were excluded from the experiment. After 5 
weeks, mice were sacrificed and the regional lymph nodes collected and examined carefully for evidence of tumor infiltration. In all studies, samples of each tumor were taken for cryostat sections or fixed immediately in Bouin's fixative for 4 hours and processed by standard paraffin embedding. Data were derived from three independent experiments in the case of the antibody treatments and from two independent experiments in the case of the surgical excision.

\section{Immunohistochemistry}

Fresh tissue sections were fixed in cold acetone, and the endogenous peroxidase activity and non-specific binding then blocked by treatment with $0.5 \% \mathrm{H}_{2} \mathrm{O}_{2}$ and normal serum (DakoCytomation, Carpinteria, CA), respectively. Sections were then incubated with primary antibodies to either CD31 (biotinylated rat anti-mouse, BioLegend, San Diego, CA) or a rabbit antibody to mouse LYVE-1 (R \& D Systems, Minneapolis, MN) overnight at $4^{\circ} \mathrm{C}$. After washing, sections were incubated with secondary antibodies/reagents, swine anti-rabbit immunoglobulins/HRP (DAKO, Glostrup, Denmark) or Streptavidin-HRP (Perkin Elmer Life Sciences, Boston, MA) each diluted 1:200, according to the species of primary antibodies, for 60 minutes. Sections were washed again and then color visualized with DAB chromogen (Vector Laboratories, Burlingame, CA). In some cases sections were stained with D2-40 (Vector Laboratories) to detect podoplanin. The endothelia detected by antibodies to PECAM-1 and LYVE-1 were quantified from sections by determining the number of stained pixels in eight randomly selected high-powered fields containing stained vessels (magnification $\times 40$ ). Computer-assisted morphometric analysis of digital images was performed using Corel Draw (Version 11.0 software, Corel Corporation, Ottawa, Canada). Total vessel area 
was calculated per tumor, using five different sections of each tumor and eight randomly selected fields per section, and data were expressed as the average vessel area of all tumors in that group.

\section{Immunofluorescence Staining}

Immunofluorescence double staining was performed on $6 \mu \mathrm{m}$ thick cryostat sections, rehydrated in mouse tonicity-PBS and blocked in normal serum (DakoCytomation, Carpinteria, CA). Primary antibodies including rabbit anti-LYVE-1, rat biotin anti-CD31 antibody (BioLegend, San Diego, CA), goat anti-VEGFR-2 and goat anti-VEGFR-3 antibodies (R\&D Systems, Minneapolis, MN) were applied overnight at $4^{\circ}$ C. Secondary chicken anti-goat Alexa 594 and donkey anti-rabbit Alexa 488 antibodies for LYVE-1-combined immunostaining or donkey anti-goat Alexa 594 and mouse anti-biotin Alexa 488 antibodies for CD31-combined immunostaining (Molecular Probes, Eugene, OR) were incubated separately for 60 minutes at room temperature. Sections were mounted in SlowFade antifade reagent (Molecular Probes). Sections were analyzed by fluorescence microscopy using a Nikon TE2000-E microscope (Nikon, Melville, NY) equipped with a DP70 Digital Camera (Olympus Australia Pty. Ltd., Melbourne, Australia).

\section{Human Breast Cancer Samples}

Primary human breast cancer tissue $(n=22)$ was obtained at the time of surgical resection and processed by standard histopathological techniques. Tumors were classified as being either "large" $(>30 \mathrm{~mm})$ or small $(<20 \mathrm{~mm})$ in diameter. These tumors were then classified according to lymph node status, being either lymph node negative (LN-) or lymph node positive ( $\mathrm{LN}+)$. Lymph node status was determined by a 
combination of sentinel lymph node biopsy and subsequent histopathology. From these groups, tumors were sub-classified according to an expected behavior pattern (being either a Large primary tumor with a positive lymph node status $\mathrm{LN}+, \mathrm{n}=5$; or a small primary tumor with a negative lymph node status, $\mathrm{LN}-, \mathrm{n}=6$ ) or to an unexpected behavior pattern (large primary tumor and negative lymph node status, $\mathrm{LN}-, \mathrm{n}=5$; small primary tumor and a positive lymph node status, $\mathrm{LN}+, \mathrm{n}=6$ ). The location, diameter and nature of lymphatic vessels in the center and periphery of these tumors were then analysed and quantified.

\section{Lymphatic Vessel Analysis in Human Tissue}

Immunohistochemical staining of human breast cancer sections was performed using the D2-40 monoclonal antibody which recognizes podoplanin (Vector Labs Inc, CA, USA). Sections were stained using a robotic immunohistochemistry workstation (IHC robot, Department of Anatomical Pathology, Royal Melbourne Hospital) and stained sections were quantified in a blinded manner using a $10 \mathrm{x}$ objective field. Each lesion was divided into different regions for quantification of lymphatic vessel location: surrounding skin, peripheral tumor (skin edge), peripheral tumor (deep edge) and central tumor regions. Control tissue derived from normal human skin and from areas of 8-12 month old scar tissue were used to analyse the lymphatic vessels in a normal and pathological context, respectively. Lymphatic vessel density (LVD) was defined as the number of podoplanin ${ }^{+}$vessels per $10 \mathrm{x}$ objective field or per $\mathrm{mm}^{2}$ as described previously.[37]

\section{Statistical Analysis}

The statistical differences for tumor size and quantitation of LYVE-1 and CD31 
immunostaining were analyzed by the Mann-Whitney $U$ test. Differences in the incidence of lymph node metastases between groups were analyzed by the $\chi^{2}$ test. $\mathrm{P}<0.05$ was considered statistically significant.

\section{Results}

Tumor Spread to Regional Lymph Nodes is an Early Event in Tumors Expressing VEGF-D

In order to characterize the timing and vessel dependency of lymphogenous metastasis, we used a previously characterized model of VEGF-D-induced tumor growth and metastasis. In this model, employing human 293EBNA-1 cells, expression of VEGF-D induces both angiogenesis and lymphangiogenesis in the primary tumor, and spread to regional lymph nodes[18]. The log-phase growth of the primary tumors, supported by angiogenesis, occurs between day 14 and 30. By surgical excision of the primary tumors on day 7 , i.e. 7 days before the onset of log-phase growth of the tumors, we explored if metastatic spread to lymph nodes occured before the major phase of primary tumor growth (Figure 1A). Macroscopic and histological examination revealed that 25-50\% of mice had tumor cells in the regional lymph node 5 weeks after surgical removal of the primary tumors (Figure 1B), indicating that metastasis can occur as an early step in tumor development. This observation parallels human cancer in which lymphogenous metastasis is seen as an early and common event in the natural history of many human cancers.

Anti-VEGFR-2 Treatment Prevents VEGF-D-Mediated Tumor Growth but is less Effective at Blocking Metastasis 
In this study, tumors expressing VEGF-D grew more rapidly than those transfected with an expression vector lacking VEGF-D sequence (APEX-3 control tumors, Figure 1C), as previously demonstrated[18], presumably due to angiogenesis driven by VEGF-D. Most VEGF-D-positive tumor-bearing mice also had tumor cell metastasis in the ipsilateral axillary lymph nodes (Figure 2A, Table 1). Treatment of VEGF-D-positive tumors using the anti-VEGFR-2 antibody DC101, strongly inhibited primary tumor growth (Figure 1C), and DC101-treated mice had small (less than $5 \mathrm{~mm}$ diameter) avascular tumors with necrotic areas, or in some cases no significant tumors at all (Figure 2E). However, despite the significant inhibition of primary tumor growth seen in the DC101-treated mice, 9 of $26(35 \%)$ mice had metastases in ipsilateral axillary or inguinal lymph nodes (Table 1). Some mice with barely detectable primary tumors had metastatic tumor cells in the local lymph node (Fig. 2L). While the involved nodes in untreated VEGF-D-positive mice were predominantly replaced with tumor cells (Fig. 2J), metastatic lymph nodes from DC101-treated VEGF-D-positive mice were generally only partially occupied by tumor cells (Fig. 2L). These findings indicate that anti-VEGFR-2 treatment was very effective in restricting the growth of the primary tumor, but less effective in inhibiting lymph node metastasis.

\section{Anti-VEGFR-3 Treatment Inhibits both Tumor Growth and Metastasis}

In contrast to DC101, treatment of VEGF-D-positive tumors with the VEGFR-3-specific monoclonal antibody mF4-31C1 only partially reduced primary tumor growth (Figure 1C and 2D). This effect on tumor growth suggests that VEGFR-3 signaling makes some contribution to angiogenesis in this tumor model. The macroscopic appearance of the tumors treated with mF4-31C1 was similar to 
VEGF-D-positive tumors treated with anti-VEGF-D antibody, being quite large and containing a visible blood vascular network (Figures $2 \mathrm{C}$ and 2D). This suggests that some blood vessels were present in the tumors after treatment, in contrast to tumors treated with DC101. Significantly, lymph node metastasis only occurred in one of 22 mice $(5 \%)$ treated with $\mathrm{mF} 4-31 \mathrm{C} 1$, in comparison to $84 \%$ of vehicle-treated VEGF-D-positive mice, indicating that the VEGFR-3 signalling pathway plays a major role in driving lymph node metastasis in this model (Table 1). Further, metastases that were detected in the lymph node of the only mouse treated with $\mathrm{mF} 4-31 \mathrm{C} 1$ that developed LN metastasis occupied a very small area of the node (Figure 2K), suggesting that the anti-VEGFR-3 antibody reduced the overall extent of lymphatic metastasis observed at the time of lymph node collection. Therefore, while targeting VEGFR-3 had a moderate impact on primary tumor growth, it was very effective (and far more effective than targeting VEGFR-2) at reducing lymphatic metastasis.

\section{The Combination of Anti-VEGFR-2 and Anti-VEGFR-3 Antibodies is the Most Effective Anti-metastatic Treatment for a VEGF-D-secreting Tumor}

Combined treatment with DC101 and mF4-31C1 antibodies was most effective in preventing both tumor growth and in preventing (or inhibiting) lymph node metastasis (Figure 1C and Table 1). Tumors treated with the DC101 + mF4-31C1 combination produced either very small or undetectable primary tumors that were macroscopically similar to those treated with DC101 alone (Figure 2F). Furthermore, lymph node metastasis was completely inhibited in this group, the combination treatment being more effective than either antibody alone (Table 1).

\section{Effects of Targeting VEGFR-2 and VEGFR-3 on Blood Vessels and Lymphatics}


We hypothesized that changes to tumor vessels induced by treatment with anti-VEGFR-2 and anti-VEGFR-3 antibodies may underlie the alterations in primary tumor growth and lymphogenous metastasis observed experimentally. It was noted that VEGFR-2 and VEGFR-3 are expressed on blood and lymphatic vessels in these tumors (data not shown). VEGF-D-positive vehicle-treated tumors exhibited an abundance of intratumoral LYVE-1-positive lymphatic vessels, which exhibited weak PECAM-1 staining, and abundant blood vessels strongly stained for PECAM-1 were also observed (Figure 3A and B). In contrast, APEX-3 control tumors contained no intratumoral LYVE-1-positive lymphatic vessels and few PECAM-1-positive blood vessels (Figure 3C and D). Treatment of VEGF-D-positive tumors with either VD-1 (anti-VEGF-D) or mF4-31C1 (anti-VEGFR-3) resulted in a substantial reduction of LYVE-1-positive lymphatic vessels (Figure 3E and G). In contrast, treatment with DC101 (anti-VEGFR-2) resulted in tumors with substantially reduced levels of PECAM-1-positive blood vessels, but prominent LYVE-1-positive lymphatic vessels persisted (Figure 3I and J). Quantification of staining patterns of LYVE-1-positive and PECAM-1-positive vessels in the various control and treatment groups demonstrated a contrasting effect of anti-VEGFR-2 and anti-VEGFR-3 treatment (Figure 4B and C). Anti-VEGFR-2 caused a substantial reduction in PECAM-1-positive vessels, which correlated with reduced primary tumor size (Figure 4A). However, the density of lymphatic vessels increased in this group, indicating that the lymphatics essentially remained intact after anti-VEGFR2 treatment (Figure 4B). In contrast, mF4-31C1 treatment resulted in a significant reduction of LYVE-1-positive lymphatic vessels (Figure 4B) while PECAM-1-positive vessels (Figure 4C) and primary tumor volume (Figure 4A) were not as reduced as for the DC101 treatment group. Further, this 
reduction in LYVE-1-positive vessels in the group treated with $\mathrm{mF} 4-31 \mathrm{C} 1$ was matched with a reduced rate of lymph node metastasis. Therefore we conclude that while anti-VEGFR-2 treatment could dramatically reduce the growth of the primary tumor through reduction of tumor blood vessels (i.e. prevention of angiogenesis), LYVE-1-positive lymphatic vessels (i.e. lymphangiogenesis) were not significantly affected by this treatment, hence the effect on lymphatic metastasis was only moderate. Conversely, anti-VEGFR-3 treatment was less able to reduce the growth of the primary tumor (Figure 4A), due to a less profound effect on the abundance of PECAM-1-positive blood vessels (Figure 4C), but was able to eliminate LYVE-1-positive lymphatic vessels (Figure 4B), and thereby result in dramatic reduction in the rate of regional nodal spread (Table 1). These data also suggest that whereas tumor size is in part determined by angiogenesis, lymph node metastasis correlates better with lymphatic vessel density.

\section{Lymphatic Vessel Density (LVD), not Tumor Size, Predicts the Outcome of some Breast Cancers}

To confirm the experimental finding of an association between high LVD and lymphatic metastasis, we examined a series of primary human breast cancers to determine if LVD in human tumors represented an independent determinant of metastasis, irrespective of tumor size and angiogenesis (Figure 5). Surgically-excised primary human breast cancers were classified as either being "large", >30 mm, or "small", <20 mm, in diameter. A $1 \mathrm{~cm}$ differential between the diameters of the two groups was chosen to allow a clear distinction between the sample sets. These tumors were then subdivided according to the lymph node status of the patients. The four groups were $\mathrm{T}+$ (large 
tumor, lymph node involved); T- (large tumor, lymph node uninvolved); t+ (small tumor, lymph node involved); t- (small tumor, lymph node uninvolved). Using D2-40 as a marker of lymphatic vessels, the density of these vessels in the intratumoral and peritumoral regions were determined (Figure 5A-E). Analysis of the average LVD across all tumor types showed little variation between intratumoral, peritumoral or total LVD (Figure 5F). In contrast, when the groups separated according to whether they fall into $\mathrm{T}+, \mathrm{T}-, \mathrm{t}+, \mathrm{t}$ - tumor categories were analysed, differences in LVD relative to the metastatic potential and size of the primary tumors were observed. Small lymph node-positive cancers $(\mathrm{t}+)$ had higher peritumoral, intratumoral and total LVD compared to the larger tumor groups, whether metastatic or not, and higher LVDs than the non-metastatic tumors of the same size (t-) (Figure 5F-I). In contrast, large lymph node positive cancers $(\mathrm{T}+)$ were not significantly different from their large tumor, lymph node-negative counterparts (T-) (Figure 5F-I). These data suggest that the LVD in small breast cancers may be associated with a more aggressive phenotype, and be a marker of those small cancers that are most likely to have lymph node metastasis at presentation. These results are consistent with our observations from the preclinical experimental tumor model that LVD, more so than angiogenesis-driven primary tumor size, is a key determinant of metastatic spread to lymph nodes.

\section{Discussion}

In the present study, we examined the contribution of blood and lymphatic vessels to primary tumor growth and lymphatic spread of tumors using a metastatic mouse tumor model and a series of primary human breast cancers. Using antibodies specific to key 
receptors for growth factors involved in angiogenesis and lymphagiogenesis, we demonstrated the contribution that VEGFR-2-mediated signaling may play in tumor angiogenesis and promoting tumor growth, and of VEGFR-3-mediated signaling for lymphangiogenesis and lymphatic metastasis (summarized in Figure 6). The key role for VEGFR-3 signaling in lymphatic metastasis is supported by studies in other rodent tumor models targeting this receptor [38-41]. The potential clinical relevance of our findings was supported by analysis of primary human breast cancers in which metastasis to regional lymph nodes correlated with lymphatic vessel density rather than tumor size. Our experiments involving surgical excision of the primary tumor indicated that lymphatic metastasis can occur when the primary tumor is very small. These insights may help in the design of anti-angiogenic and/or anti-lymphangiogenic treatment regimens for cancer therapy[42], particularly in defining the time window during which these treatments may be most effective. More specifically, our data suggest that anti-lymphangiogenic approaches designed to restrict tumor metastasis should be undertaken as early as possible to maximize the likelihood of restricting tumor dissemination.

The anti-VEGFR-3 antibody mF4-31C1 restricted tumor growth in our study, in addition to blocking lymph node metastasis. The anti-growth effect was presumably due to reduced signalling via VEGFR-3 homodimers or VEGFR-2/VEGFR-3 heterodimers localized on blood vessels. VEGFR-2/VEGFR-3 heterodimers were recently reported to be localized in filopodia of leading tip cells on angiogenic sprouts, and are thought to positively regulate angiogenic sprouting [43]. Further, it has been shown that blocking VEGFR-3 signalling results in decreased sprouting, vascular density, vessel branching 
and endothelial cell proliferation in a range of mouse angiogenesis models [44], as well as restricting primary tumor growth [45]. Other groups have shown that VEGF-D has a potential role as an autocrine growth factor in breast cancer[46], suggesting a mechanism whereby expression of VEGF-D affects directly the tumor cells. We have found that the expression of VEGF-D and other VEGF family growth factors do not alter the growth characteristics of 293 cells in vitro suggesting that such an autocrine loop does not exist in this tumor model[18].

Our data on the contribution of VEGFR-2 and VEGFR-3 to lymph node metastasis are consistent with our recently published study, using the same tumor model, showing that the collecting lymphatic vessels draining primary tumors can contribute to metastatic spread in a VEGFR-2 and VEGFR-3 dependent manner [17]. In the study reported here, DC101 demonstrated efficacy in preventing angiogenesis in, and growth of, the primary tumour. DC101 was however less effective in preventing metastasis. The reduction of $65 \%$ in regional LN spread in DC101-treated tumors could be due to a number of effects. VEGFR-2 is expressed to some degree on small lymphatic vessels and therefore could provide a target here, similar to the more highly expressed VEGFR-3. We have shown in another study that inhibiting VEGFR-2 on collecting lymphatic vessels likely leads to reduced transport of tumor cells [17]. Further anti-VEGFR-2 Mab could be working at sites of metastasis to reduce the proliferation of any metastatic cells via inhibition of angiogenesis thus reducing the opportunity to detect tumor involved lymph nodes. Importantly, the difference between the DC101 and mF4-31C1 Mabs in their ability to inhibit $\mathrm{LN}$ metastasis (65\% vs $95 \%$ inhibition) appears to reside in the ability of anti-VEGFR-3 treatment to inhibit the effects of the lymphangiogenic growth factor 
on new or preexisting lymphatic vessels within and around the primary tumor. This conclusion is supported by the analysis of primary human breast cancers of different sizes in which tumors with high peritumoral LVD, rather than greater size, were more likely to metastasise. It is interesting that experimental data using inhibitors of the VEGF pathway in tumor models indicated increased local invasion and metastatic spread[47, 48]. This was seen after short-term treatment with an anti-angiogenic inhibitor in one case, however, others have suggested that this may be highly dependent on the class of drugs being investigated[49-51]. In our study we did not observe increased local invasion in response to anti-VEGFR-2 or anti-VEGFR-3 Mabs. The proposed pro-metastatic effect of anti-angiogenic reagents requires further evaluation at the experimental and clinical levels.

Clinically, the contributions of blood and lymphatic vessels, both new and preexisting, to the natural history of a tumor are not clearly defined [52]. Tumor growth, regional and distant organ metastasis, and ultimately patient outcome are all, in part, dependent on the function of blood and lymphatic vessels within and around a tumor [33]. While blood and lymphatic vessels may contribute to the escape of tumor cells from the primary tumor, they are also involved in establishing routes for delivery of nutrition and removal of cellular waste, thus facilitating primary tumor growth. Dissecting the roles that distinct vessel types play in the processes of tumor growth and metastasis will be important to assess optimal therapeutic approaches for targeting tumor angiogenesis and lymphangiogenesis. For example, a new area of active investigation is the analysis of distinct lymphatic vessel subtypes in cancer metastasis, and the molecular control of lymphatics involved in the uptake and transport of metastasizing tumor cells [17]. 
Our findings from primary human breast cancers, that metastasis to regional lymph nodes correlated with lymphatic vessel density rather than tumor size, may have clinical implications. They suggest that high LVD in and around a primary breast cancer may have prognostic and therapeutic significance. From a prognostic point of view, lymph node involvement has long been recognized as the most important prognostic factor for patients with early breast cancer. We have found an association between LVD and nodal involvement. Further studies will be required to determine whether LVD is an independent prognostic factor, or indeed if it is a more useful factor than lymph node involvement. Also, further study of in vivo orthotopic models for diseases such as breast cancer[17], combined with biomarker data from relevant human cancers will enable the connection between the 293 model and the primary tumor data identified in this study to be fully validated.

The management of axillary nodal metastasis has become more selective - initially with sentinel node biopsy and more recently with the selective approach to complete axillary dissection in the case of positive sentinel nodes[53]. The next logical step is a selective approach to any axillary nodal assessment. Our data suggest that patients whose cancer has high lymphatic vessel density are at substantially higher risk of nodal involvement and of more extensive nodal involvement. Caution would be required before reducing the extent of nodal surgery in these patients. Furthermore, recent genomic mapping of pancreatic cancer metastases has provided evidence for a series of mutational events and clonal evolution during the metastatic process, such that metastasis should be seen as a multi-step process, not a single release of cells from only the primary tumor[2]. Hence 
the nature of the metastatic cell can be a more important issue than the size of the tumor. Together, these findings indicate that the primary tumor need not have reached a significant size for initial lymph node metastasis to take place, and challenges the idea that tumors of a small size should be considered cured after surgical excision.

Collectively, our data suggest that combination treatment with anti-VEGFR-2 and anti-VEGFR-3 antibodies most thoroughly restricts primary tumor growth and lymph node metastasis. Both experimental and histopathological data show that VEGFR-3 signaling in lymphatic endothelial cells, and LVD, are key parameters relating to metastatic spread, whereas angiogenic signaling contributes principally to tumor growth. These results are consistent with recent findings that adjuvant trials with anti-angiogenic agents such as the anti-VEGF-A antibody Bevacizumab seem to have little effect on the long-term survival of cancer patients, while providing initial reduction in tumor burden for some patients[8]. Further characterization of the vascular effects of specific signaling pathways should help us resolve the contribution of different vessel types to the complex process of tumor progression. 
Table 1. Effects of Monoclonal Antibody Treatment on Lymph Node Metastasis

\begin{tabular}{|c|c|c|c|}
\hline Tumor type & Antibody Treatment & $\begin{array}{l}\text { Proportion of Mice } \\
\text { with Lymph Node } \\
\text { Metastasis }^{\mathrm{a}}\end{array}$ & $\begin{array}{l}\text { Number of Involved } \\
\text { Regional Lymph } \\
\text { Nodes in all Mice }^{\mathrm{a}}\end{array}$ \\
\hline VEGF-D $^{+}$ & Vehicle & $21 / 25(84 \%)$ & 27 \\
\hline APEX-3 & Vehicle & $0 / 25(0 \%)^{b}$ & 0 \\
\hline VEGF-D $^{+}$ & VD1 & $3 / 27(11 \%)^{\mathrm{b}, \mathrm{d}}$ & 3 \\
\hline VEGF-D $^{+}$ & $\mathrm{mF} 4-31 \mathrm{C} 1$ & $1 / 22(5 \%)^{b, d}$ & 1 \\
\hline VEGF-D $^{+}$ & DC101 & $9 / 26(35 \%)^{c}$ & 10 \\
\hline VEGF-D $^{+}$ & $\mathrm{mF} 4-31 \mathrm{C} 1+\mathrm{DC} 101$ & $0 / 25(0 \%)^{b, d}$ & 0 \\
\hline
\end{tabular}

${ }^{\mathrm{a}}$ Numbers are totals derived from three experiments

${ }^{\mathrm{b}} \mathrm{P}<0.0001$ compared with VEGF-D+ tumors treated by vehicle using Mann-Whitney U test ${ }^{\mathrm{c}} \mathrm{P}<0.001$ compared with VEGF-D+ tumors treated by vehicle using Mann-Whitney U test ${ }^{\mathrm{d}} \mathrm{P}<0.05$ compared with DC101-treated tumors by the Mann-Whitney U test 


\section{Figure Legends}

Figure 1. Growth of VEGF-D over-expressing human tumor xenografts in the skin of SCID/NOD mice, and inhibition with receptor- and growth factor-specific monoclonal antibodies. (A) Tumors expressing the lymphangiogenic growth factor VEGF-D grow slowly in the initial phase (Days 1-14) of their establishment as skin xenografts. Tumors then undergo an exponential growth phase promoted by angiogenesis (shaded area). Surgical removal of the primary tumors was performed at day 7 (indicated by the inverted triangle), in the early phase of tumor growth, to establish if lymph node metastasis occurs during this initial phase of tumor establishment. (B) Section of tumor-involved regional lymph node from mouse in which the primary tumor had been surgically removed. The dotted boundary indicates region of tumor cells (T). (C) Treatment of VEGF-D-positive tumors with monoclonal antibodies to VEGF-D (VD1), VEGFR-3 (mF4-31C1), VEGFR-2 (DC101) or to both VEGFR-2 and VEGFR-3 (mF4-31C1 + DC101). Control tumors include VEGF-D-positive tumors treated with vehicle alone (VEGF-D Control), and a non-metastatic cell line expressing only the pAPEX-3 vector (APEX-3 Control) also treated with vehicle. Groups were followed until they reached a maximum size allowable under ethics approval. Data are presented as mean +/- SEM.

Figure 2. Macroscopic and histological appearance of primary tumors and regional lymph nodes. Mice with VEGF-D-positive tumors (A, C-F) or APEX-3 Control tumors (B) were examined post-mortem at the completion of the treatment regime. Panels show the ventral skin (Sk) reflected back to reveal the tumor (designated $\mathrm{T}$ ), the major 
collecting lymphatic vessel (CLV, arrowed, and prominently displayed in A) and the area of the axillary lymph node $(\mathrm{Ax})$ at the anterior end of the mouse. The head and tail of the mouse is indicated for orientation, and the intact peritoneal cavity sits in the lower part of the images. (A) VEGF-D-positive tumor treated with vehicle, (B) APEX tumor, (C) VEGF-D-positive tumor treated with anti-VEGF-D Mab VD1, (D) VEGF-D-positive tumor treated with anti-VEGFR-3 Mab mF4-31C1, (E) VEGF-D-positive tumor treated with anti-VEGFR-2 Mab DC101 and (F) VEGF-D-positive tumor treated with a combination of anti-VEGFR-2 and anti-VEGFR-3 Mabs. In E \& F small avascular tumors are indicated by arrows. Comparison of primary tumor tissue from control VEGF-D-positive tumors treated with vehicle $(\mathrm{G})$ and VEGF-D-positive tumors treated with anti-VEGFR-2 Mab $(\mathrm{H})$ shows that anti-VEGFR-2 treatment induces areas of substantial necrosis (designated N) which are not present in the compact cellular mass of the vehicle-treated tumor. Regional lymph nodes (axillary and superficial inguinal) were removed from mice at post-mortem and examined by histology for the presence of tumor cells (I-L). Normal lymph nodes with no tumor cells (I) were removed from APEX-3 tumor bearing mice whereas lymph nodes from VEGF-D-positive vehicle-treated mice $(\mathrm{J})$ had fully invaded lymph nodes with minimal normal tissue present. Lymph nodes from VEGF-D-positive mice treated with anti-VEGFR-3 typically had no tumor cells or very limited metastases (K) whereas those treated with anti-VEGFR-2 (L) had variable sized metastases (indicated by dotted lines).

Figure 3. Immunohistochemical analysis of primary tumors for blood and lymphatic vessels. Sections of primary tumor tissue were stained with antibodies to 
LYVE-1 (A, C, E, G, I, K) and PECAM-1 (B, D, F, H, J, L) and detected using a DAB substrate and hematoxylin counter staining. Tumors analysed were VEGF-D (A, B) and APEX3 vehicle control (C, D) tumors, and VEGF-D tumors treated with antibodies to VEGF-D (VD1) (E, F); mouse VEGFR-3 (mF4-31C1) $(\mathrm{G}, \mathrm{H})$; mouse VEGFR-2 (DC101), (I, J); mouse VEGFR-3 and VEGFR-2 (mF4-31C1 + DC101) (K, L). "n” in panel $\mathrm{J}$ denotes necrotic regions. Arrows in $\mathrm{F}, \mathrm{H}$ and $\mathrm{L}$ denote the remnants of PECAM-1+ vessels.

Figure 4. Quantification of tumor growth and vessel formation. Primary tumors were excised from mice after treatment with Mabs for 5 weeks, and mean tumor volumes determined (A). The groups were 293-VEGF-D tumors treated with vehicle (PBS), anti-VEGF-D Mab (VD1), anti-mouse VEGFR-3 Mab (mF4-31C1, abbreviation mF4 used), anti-mouse VEGFR-2 Mab (DC101) and the combination of anti-mouse VEGFR-3 and VEGFR-2 Mabs (mF4-31C1 + DC101). Vehicle-treated APEX3 tumors were included as an extra control. The endothelium of lymphatic vessels in the primary tumors was quantitated by immunostaining for LYVE-1 (B) and the endothelium of blood vessels by immunostaining for PECAM-1 (C). The values represent the average area of sections stained by either LYVE-1 or PECAM-1 antibodies and are expressed as the number of pixels $\left(\times 10^{4}\right)$ per image that fall above a threshold for positive staining. The statistical significance of differences between study groups was assessed by the Mann-Whitney U test; * $\mathrm{p}<0.05$.

\section{Figure 5. Analysis of lymphatic vessel distribution in human breast tumors.}

Sections of human breast tumors, normal human skin and scar tissue were used to 
determine lymphatic vessel density. Tumors were analysed in three layers: i) peripheral tumor at the skin edge - representing the part of the tumor towards the skin of the patient; ii) peripheral tumor at the deep edge - representing the edge of the tumor furthest from the skin; iii) central tumor defined as the middle third of the tumor between the other two layers (A). Areas of tumors corresponding to $1 \mathrm{X} 10$ objective fields (see Materials and Methods) were used to quantitate LVD in these regions (B). Normal skin samples (C) and resolved scar tissue (D) were analysed for LVD as controls (x100). Sections of breast cancers were stained by immunohistochemistry for lymphatic vessels with the antibody D2-40, x40 (E). The margin of the tumor is indicated in the section by a dotted line. The LVD was determined in the peritumoral and intratumoral regions of the breast tumors, and the LVDs of these two regions were combined (designated "Total") (F). The human breast tumors were sub-divided into groups according to the size of the primary tumor and lymph node status $(\mathrm{T}+$ denotes large primary tumor and involved lymph nodes; T- large primary tumor and non-involved lymph nodes; $\mathrm{t}$ - small primary tumor and non-involved lymph nodes; $\mathrm{t}+$ small primary tumor and involved lymph nodes). These tumors were evaluated for peritumoral LVD (G), intratumoral LVD (H) and total LVD (I). Arrows indicate stained vessels. The epidermis in $(C)$ is indicated by "e". Data are presented as mean \pm SEM. Data analysed using a Students t-test. $* * \mathrm{P}<0.01, * \mathrm{P}<0.05$.

Figure 6. Schematic model of the effects of anti-VEGF receptor antibodies on primary tumor growth and metastatic spread. VEGF-D induces angiogenesis (principally via VEGFR-2 but also via VEGFR-3) and lymphangiogenesis (principally via VEGFR-3) in the primary tumor, facilitating primary tumor growth and lymph node 
metastasis, respectively (VEGF-D Control). Treatment of tumors with anti-VEGFR-3 antibody restricts tumor growth to a moderate extent, and dramatically inhibits tumor cell spread to regional lymph nodes via lymphatic vessels in and around the primary tumor (VEGF-D + Anti-VEGFR3 Mab). Treatment with anti-VEGFR-2 antibody dramatically inhibits primary tumor growth, by restricting angiogenic blood vessels in the primary tumor, but the resulting small tumors have significant metastatic capacity (VEGF-D + Anti-VEGFR-2 Mab) due to a high density of residual lymphatic vessels in and around the primary tumor. Both anti-VEGFR-2 and anti-VEGFR-3 treatments have a role in restricting the dilation of major lymphatic collectors which also inhibits the capacity for metastatic spread. The combination treatment with anti-VEGFR-3 and anti-VEGFR-2 antibodies most effectively inhibits both primary tumor growth and lymph node metastasis. The percentages shown denote the proportion of mice with involved lymph nodes as observed in the tumor model described here.

\section{Acknowledgements}

We thank You-Fang Zhang for scientific and technical help with immunohistochemistry, and the staff of the Animal Facility at the Ludwig Institute for Cancer Research for assistance with SCID/NOD mice. We also thank Anthony Burgess for helpful discussion and critical reading of this manuscript.

\section{Conflict of Interest}

SAS and MGA are on the Scientific Advisory Board of Vegenics Ltd, a wholly owned subsidiary of Circadian Technologies, are stock holders and consultants. 


\section{References}

1. Fidler IJ (2003) The pathogenesis of cancer metastasis: the 'seed and soil' hypothesis revisited. Nature RevCancer 3: 453-8

2. Campbell PJ, Yachida S, Mudie LJ, et al (2010) The patterns and dynamics of genomic instability in metastatic pancreatic cancer. Nature 467: 1109-13

3. Ferrara N (2002) VEGF and the quest for tumour angiogenesis factors. NatRevCancer 2: 795-803

4. Ferrara N, Hillan KJ, Gerber HP, et al (2004) Discovery and development of bevacizumab, an anti-VEGF antibody for treating cancer. NatRevDrug Discov 3: 391-400

5. Witte L, Hicklin DJ, Zhu Z, et al (1998) Monoclonal antibodies targeting the VEGF receptor-2 (Flk1/KDR) as an anti-angiogenic therapeutic strategy. Cancer Metastasis Rev 17: $155-61$

6. Hurwitz H, Fehrenbacher L, Novotny W, et al (2004) Bevacizumab plus irinotecan, fluorouracil, and leucovorin for metastatic colorectal cancer. New Engl J Med 350: 2335-42

7. Hurwitz HI, Fehrenbacher L, Hainsworth JD, et al (2005) Bevacizumab in combination with fluorouracil and leucovorin: an active regimen for first-line metastatic colorectal cancer. J Clin Oncol 23: 3502-8

8. Allegra CJ, Yothers G, O'Connell MJ, et al (2011) Phase III trial assessing bevacizumab in stages II and III carcinoma of the colon: results of NSABP protocol C-08. J Clin Oncol 29: 11-6

9. Joukov V, Pajusola K, Kaipainen A, et al (1996) A novel vascular endothelial growth factor, VEGF-C, is a ligand for the Flt4 (VEGFR-3) and KDR (VEGFR-2) receptor tyrosine kinases. The EMBO journal 15: 290-98

10. Achen MG, Jeltsch M, Kukk E, et al (1998) Vascular endothelial growth factor D (VEGF-D) is a ligand for the tyrosine kinases VEGF receptor 2 (Flk1) and VEGF receptor 3 (Flt4). Proc Natl Acad Sci USA 95: 548-53

11. Achen MG, Stacker SA (2012) Vascular endothelial growth factor-D:signalling mechanisms, biology and clinical relevance. Growth Factors 5: 283-96

12. Banerji S, Ni J, Wang S-X, et al (1999) LYVE-1, a new homologue of the CD44 glycoprotein, is a lymph-specific receptor for hyaluronan. J Cell Biol 144: 789-801

13. Breiteneder-Geleff S, Soleiman A, Kowalski H, et al (1999) Angiosarcomas express mixed endothelial phenotypes of blood and lymphatic capillaries. Podoplanin as a specific 
marker for lymphatic endothelium. The American journal of pathology 154: 385-94

14. Mandriota SJ, Jussila L, Jeltsch M, et al (2001) Vascular endothelial growth factor-C-mediated lymphangiogenesis promotes tumour metastasis. The EMBO journal 20: $672-82$

15. Skobe M, Hamberg LM, Hawighorst T, et al (2001) Concurrent induction of lymphangiogenesis, angiogenesis, and macrophage recruitment by vascular endothelial growth factor-C in melanoma. Am J Pathol 159: 893-903

16. Shayan R, Inder R, Karnezis T, et al (2013) Tumor location and nature of lymphatic vessels are key determinants of cancer metastasis. Clin Exp Metastasis 30: 345-56

17. Karnezis T, Shayan R, Caesar C, et al (2012) VEGF-D promotes tumor metastasis by regulating prostaglandins produced by the collecting lymphatic endothelium. Cancer Cell 21: $181-95$

18. Stacker SA, Caesar C, Baldwin ME, et al (2001) VEGF-D promotes the metastatic spread of tumor cells via the lymphatics. Nature Med 7: 186-91

19. Shayan R, Achen MG, Stacker SA (2006) Lymphatic vessels in cancer metastasis: bridging the gaps. Carcinogenesis 27: 1729-38

20. Skobe M, Hawighorst T, Jackson DG, et al (2001) Induction of tumor lymphangiogenesis by VEGF-C promotes breast cancer metastasis. Nature Med 7: 192-8

21. Kopfstein L, Veikkola T, Djonov VG, et al (2007) Distinct roles of vascular endothelial growth factor-D in lymphangiogenesis and metastasis. Am J Pathol 170: 1348-61 22. Farnsworth RH, Karnezis T, Shayan R, et al (2011) A role for bone morphogenic protein-4 in vascular endothelial growth factor-D mediated tumor growth, metastasis and vessel remodelling. Cancer Res 71: 6547-57

23. Achen MG, Roufail S, Domagala T, et al (2000) Monoclonal antibodies to vascular endothelial growth factor-D block its interactions with both VEGF receptor-2 and VEGF receptor-3. Eur J Biochem 267: 2505-15

24. Stacker SA, Williams RA, Achen MG (2004) Lymphangiogenic growth factors as markers of tumor metastasis APMIS 112: 539-49

25. Kitadai Y, Kodama M, Cho S, et al (2005) Quantitative analysis of lymphangiogenic markers for predicting metastasis of human gastric carcinoma to lymph nodes. IntJ Cancer 115: $388-92$

26. White JD, Hewett PW, Kosuge D, et al (2002) Vascular endothelial growth factor-D expression is an independent prognostic marker for survival in colorectal carcinoma. Cancer Res 62: 1669-75

27. Renyi-Vamos F, Tovari J, Fillinger J, et al (2005) Lymphangiogenesis correlates with lymph node metastasis, prognosis, and angiogenic phenotype in human non-small cell 
lung cancer. Clin Cancer Res 11: 7344-53

28. Schoppmann SF, Bayer G, Aumayr K, et al (2004) Prognostic value of lymphangiogenesis and lymphovascular invasion in invasive breast cancer. Annals of surgery 240: 306-12

29. Rubbia-Brandt L, Terris B, Giostra E, et al (2004) Lymphatic vessel density and vascular endothelial growth factor-C expression correlate with malignant behavior in human pancreatic endocrine tumors. Clin Cancer Res 10: 6919-28

30. Sipos B, Klapper W, Kruse ML, et al (2004) Expression of lymphangiogenic factors and evidence of intratumoral lymphangiogenesis in pancreatic endocrine tumors. American Journal of Pathology 165: 1187-97

31. Yokoyama Y, Charnock-Jones DS, Licence D, et al (2003) Expression of vascular endothelial growth factor (VEGF)-D and its receptor, VEGF receptor 3, as a prognostic factor in endometrial carcinoma. ClinCancer Res 9: 1361-9

32. Yokoyama Y, Charnock-Jones DS, Licence D, et al (2003) Vascular endothelial growth factor-D is an independent prognostic factor in epithelial ovarian carcinoma. $\mathrm{Br} \mathrm{J}$ Cancer 88: 237-44

33. Achen MG, McColl BK, Stacker SA (2005) Focus on lymphangiogenesis in tumor metastasis. Cancer Cell 7: 121-7

34. Debinski W, Slagle-Webb B, Achen MG, et al (2001) VEGF-D is an X-linked/AP-1 regulated putative onco-angiogen in human glioblastoma multiforme. Mol Med 7: 598-608

35. Stacker SA, Stenvers K, Caesar C, et al (1999) Biosynthesis of vascular endothelial growth factor-D involves proteolytic processing which generates non-covalent homodimers. $\mathrm{J}$ Biol Chem 274: 32127-36

36. Pytowski B, Goldman J, Persaud K, et al (2005) Complete and specific inhibition of adult lymphatic regeneration by a novel VEGFR-3 neutralizing antibody. J Natl Cancer Inst 97: $14-21$

37. Shayan R, Karnezis T, Tsantikos E, et al (2007) A system for quantifying the patterning of the lymphatic vasculature. Growth Factors 25: 417-25

38. Roberts N, Kloos B, Cassella M, et al (2006) Inhibition of VEGFR-3 activation with the antagonistic antibody more potently suppresses lymph node and distant metastases than inactivation of VEGFR-2. Cancer Res 66: 2650-7

39. He Y, Kozaki K, Karpanen T, et al (2002) Suppression of tumor lymphangiogenesis and lymph node metastasis by blocking vascular endothelial growth factor receptor 3 signaling. J Natl Cancer Inst 94: 819-25

40. He Y, Rajantie I, Pajusola K, et al (2005) Vascular endothelial cell growth factor receptor 3-mediated activation of lymphatic endothelium is crucial for tumor cell entry and 
spread via lymphatic vessels. Cancer Res 65: 4739-46

41. Burton JB, Priceman SJ, Sung JL, et al (2008) Suppression of prostate cancer nodal and systemic metastasis by blockade of the lymphangiogenic axis. Cancer Res 68: 7828-37

42. Achen MG, Mann GB, Stacker SA (2006) Targeting lymphangiogenesis to prevent tumour metastasis. BrJ Cancer 94: 1355-60

43. Nilsson I, Bahram F, Li X, et al (2010) VEGF receptor 2/-3 heterodimers detected in situ by proximity ligation on angiogenic sprouts. The EMBO journal 29: 1377-88

44. Tammela T, Zarkada G, Wallgard E, et al (2008) Blocking VEGFR-3 suppresses angiogenic sprouting and vascular network formation. Nature 454:656-60

45. Laakkonen P, Waltari M, Holopainen T, et al (2007) Vascular endothelial growth factor receptor 3 is involved in tumor angiogenesis and growth. Cancer Res 67: 593-9

46. Akahane M, Akahane T, Shah A, et al (2005) A potential role for vascular endothelial growth factor-D as an autocrine growth factor for human breast carcinoma cells. Anticancer Res 25: 701-7

47. Ebos JM, Lee CR, Cruz-Munoz W, et al (2009) Accelerated metastasis after short-term treatment with a potent inhibitor of tumor angiogenesis. Cancer Cell 15: 232-9

48. Paez-Ribes M, Allen E, Hudock J, et al (2009) Antiangiogenic therapy elicits malignant progression of tumors to increased local invasion and distant metastasis. Cancer Cell 15: 220-31

49. Singh M, Couto SS, Forrest WF, et al (2012) Anti-VEGF antibody therapy does not promote metastasis in genetically engineered mouse tumour models. J Pathol 227: 417-30

50. Chung AS, Kowanetz M, Wu X, et al (2012) Differential drug class-specific metastatic effects following treatment with a panel of angiogenesis inhibitors. J Pathol 227: 404-16

51. Stacker SA, Achen MG (2013) Where to now with the VEGF signalling pathway in cancer? Chinese Journal of Cancer Feb 19. doi: 10.5732/cjc.012.10319. [Epub ahead of print] PubMed PMID: 23419196.

52. Achen MG, Stacker SA (2008) Molecular control of lymphatic metastasis. Ann NY Acad Sci 1131: 225-34

53. Chu KU, Turner RR, Hansen NM, et al (1999) Do all patients with sentinel node metastasis from breast carcinoma need complete axillary node dissection? Ann Surg 229: $536-41$ 
A.

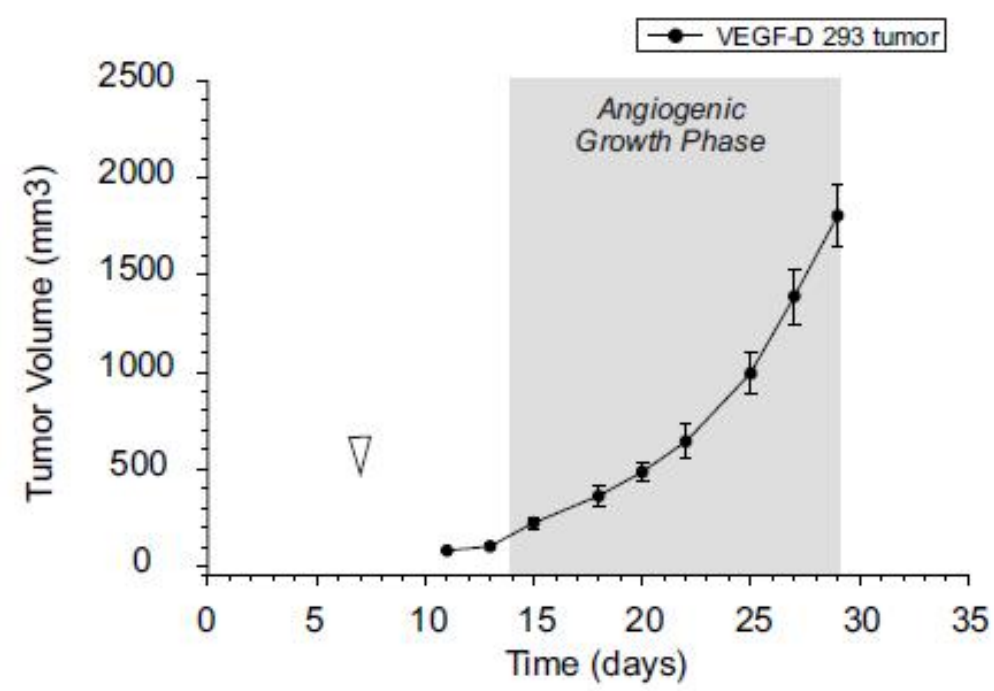

Surgical excision of tumors $(\nabla)$

B.

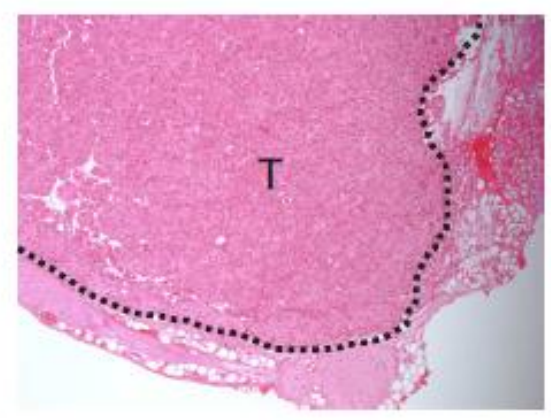

c.

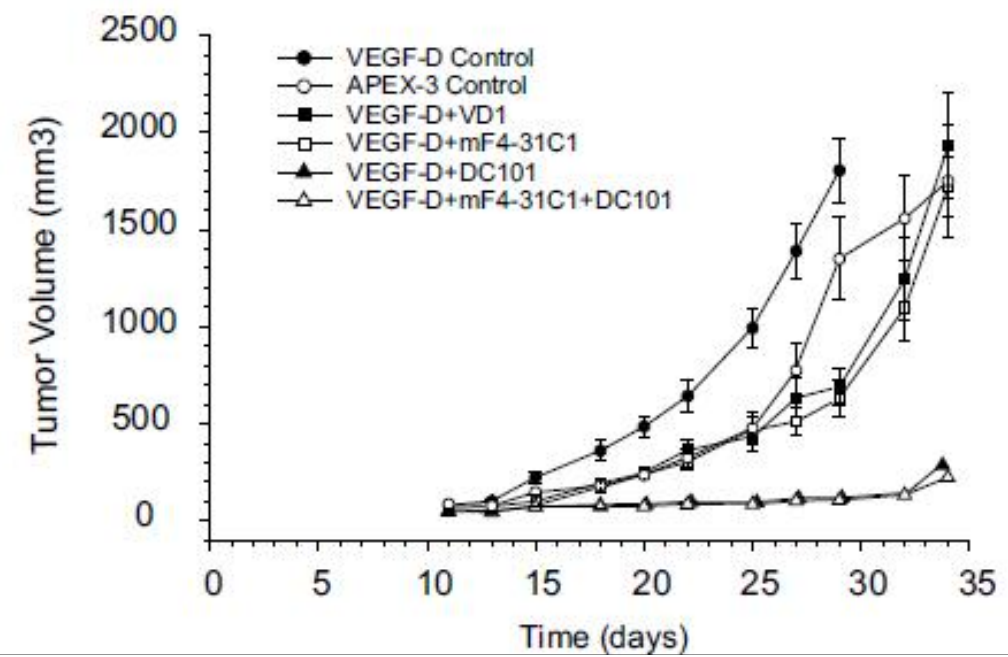




\section{Figure 2.}

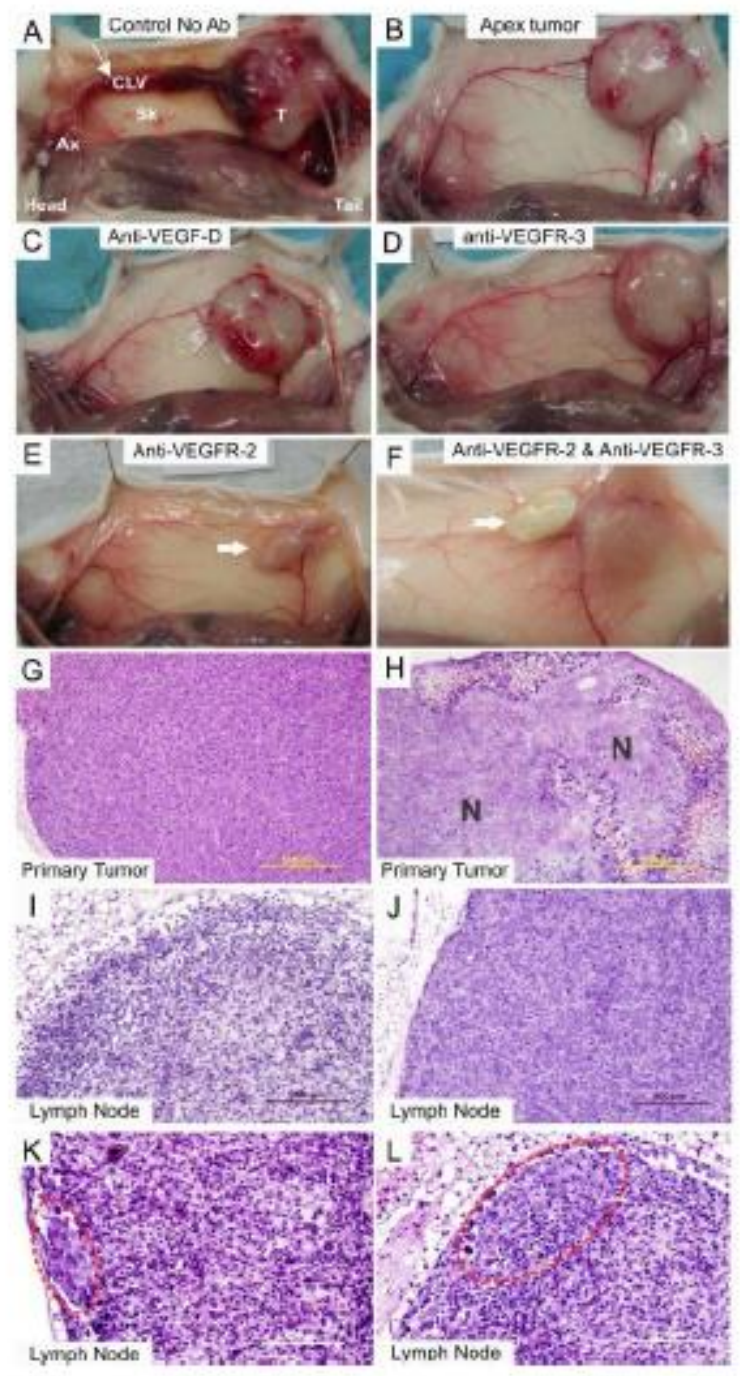


grirg.gpe 3

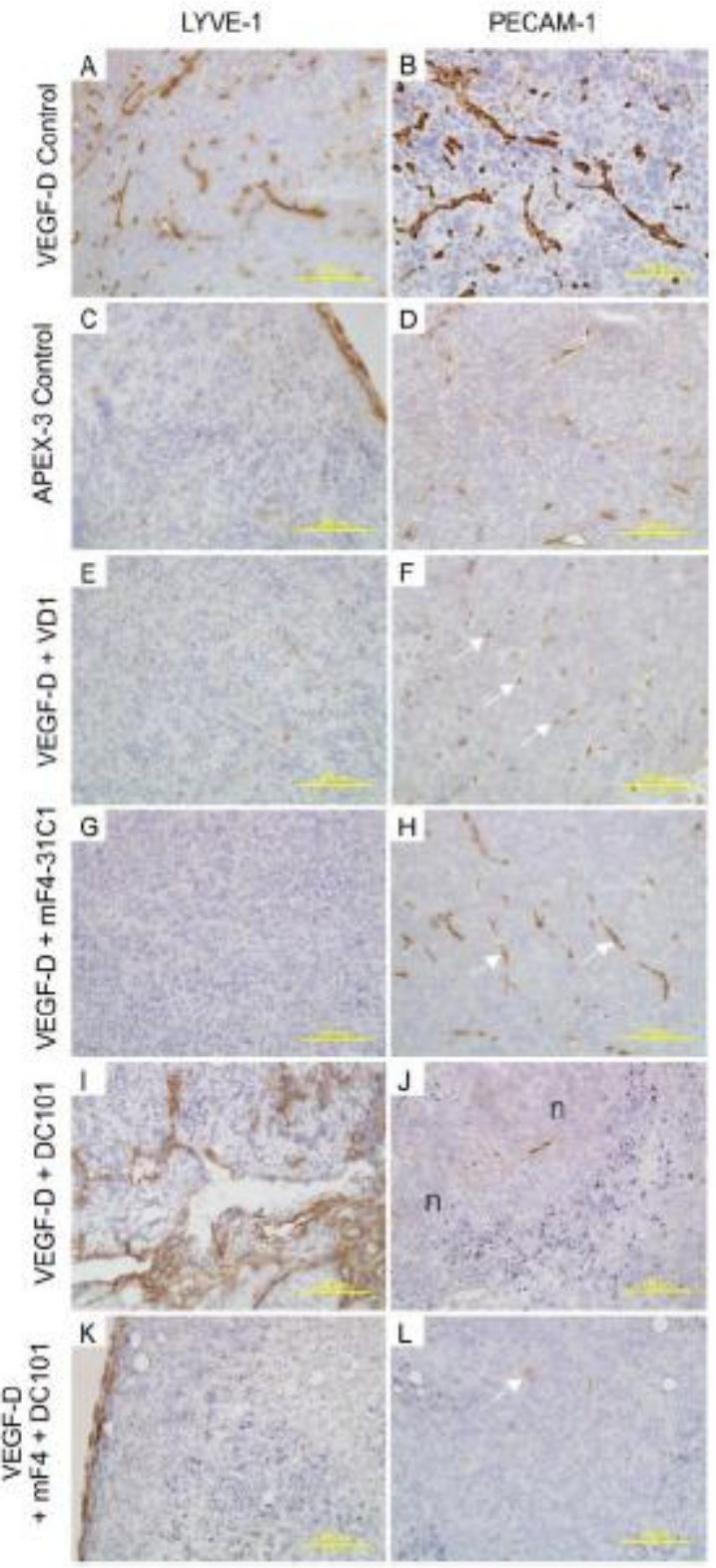


peFigure 4

A.

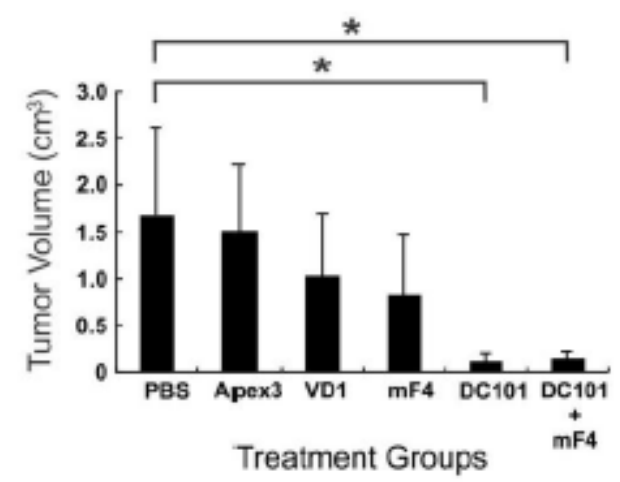

B.

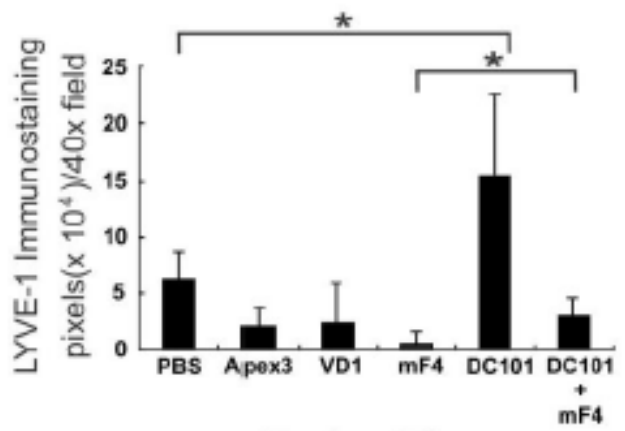

Treatment Groups

C.

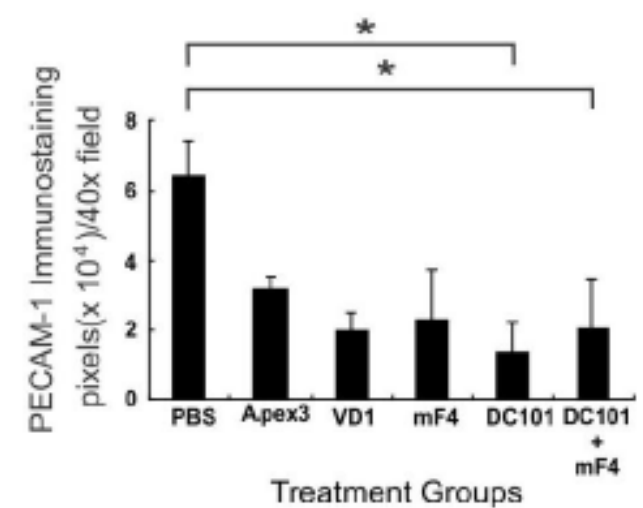




\section{Figure5.ppt Figure 5}

A.

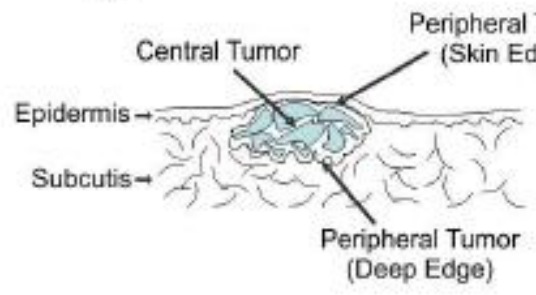

B.

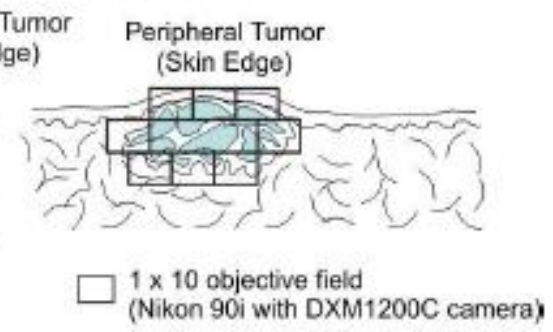

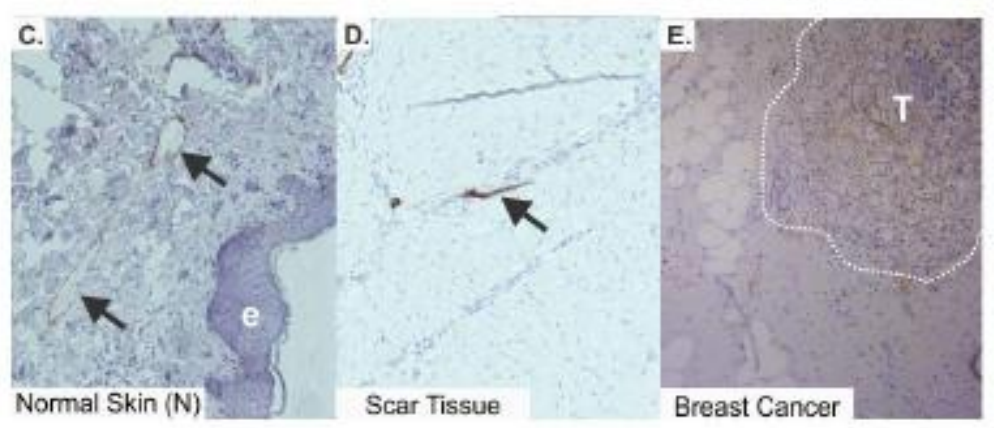

F.

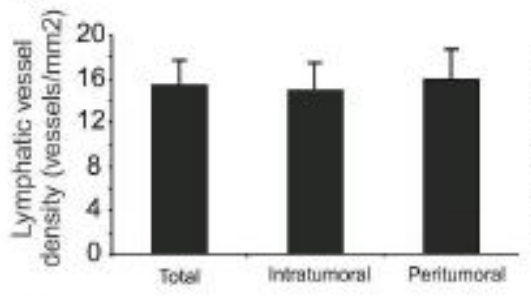

H.

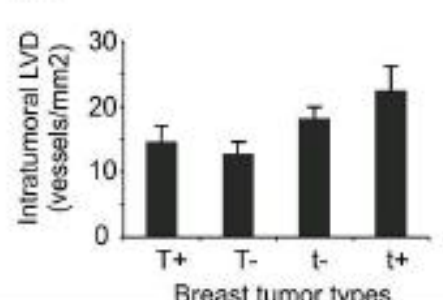

G.
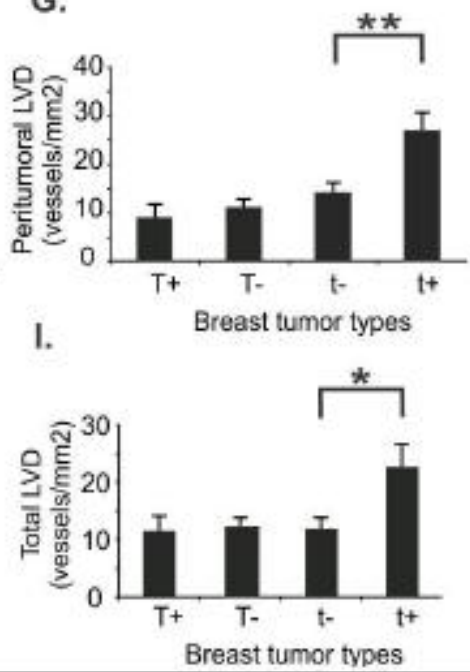
Figure 6

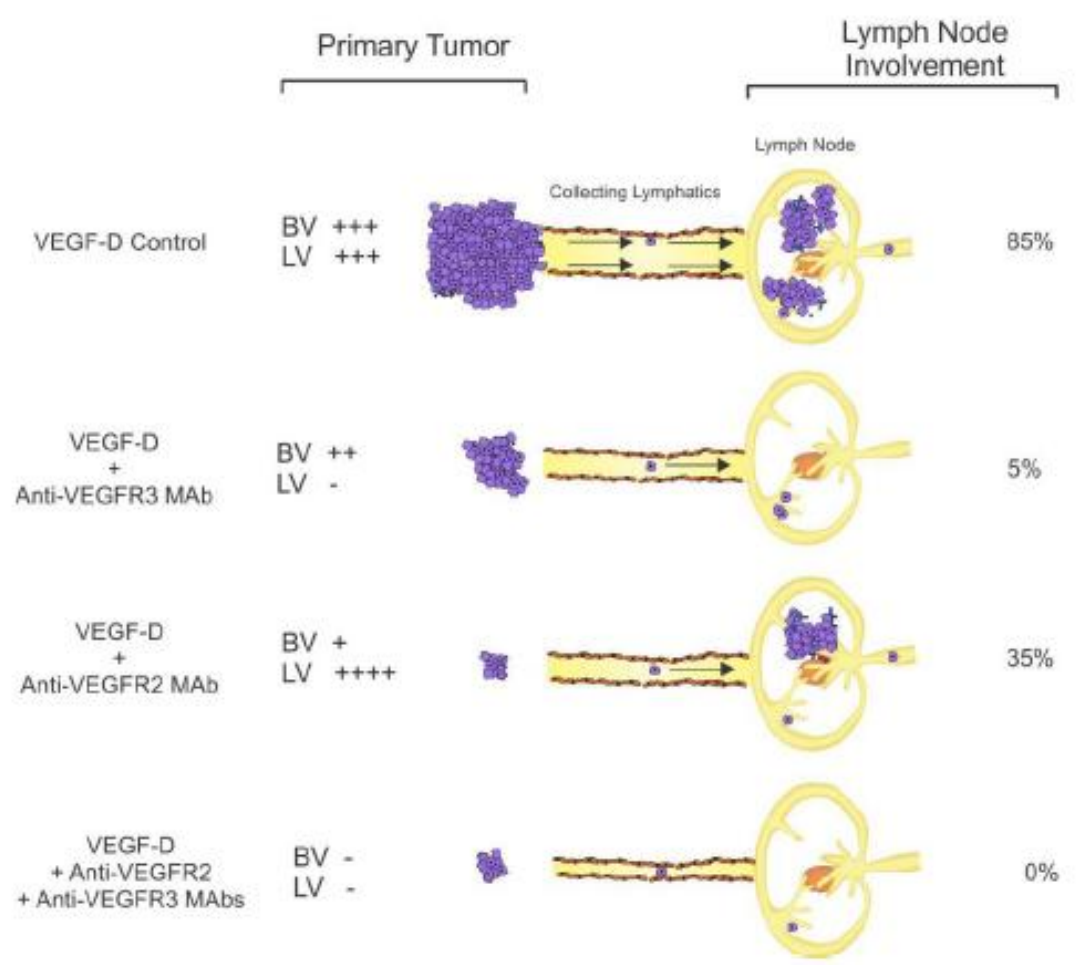

